\title{
Membrane Recycling in the Neuronal Growth Cone Revealed by FM1-43 Labeling
}

\author{
Thomas J. Diefenbach, Peter B. Guthrie, Heike Stier, Brian Billups, and S. B. Kater \\ Department of Neurobiology and Anatomy, University of Utah, Salt Lake City, Utah 84132
}

\begin{abstract}
Membrane dynamics within the chick ciliary neuronal growth cone were investigated by using the membrane-impermeant dye FM1-43. A depolarization-evoked endocytosis was observed that shared many properties with the synaptic vesicle recycling previously described at the presynaptic terminal. In addition, in the absence of depolarization a basal level of constitutive endocytotic activity was observed at $\sim 30 \%$ of the rate of evoked endocytosis. This constitutive endocytosis accounted for large amounts of membrane retrieval: the equivalent of the entire growth cone surface area could be internalized within a $30 \mathrm{~min}$ period. Endosomes generated via constitutive and evoked processes were highly mobile and could move considerable distances both within the growth cone and out to
\end{abstract}

the neurite. In addition to their different requirements for formation, evoked and constitutive endosomes displayed a significant difference in release properties. After a subsequent depolarization of labeled growth cones, evoked endosomes were released although constitutive endosomes were not released. Furthermore, treatment with latrotoxin released evoked endosomes, but not constitutive endosomes. Although the properties of evoked endosomes are highly reminiscent of synaptic vesicles, constitutive endosomes appear to be a separate pool resulting from a distinct and highly active process within the neuronal growth cone.

Key words: endocytosis; growth cone; exocytosis; FM1-43; endosome; synaptic vesicle; latrotoxin
The mechanisms underlying diverse neuronal growth cone behaviors ranging from motility to neurotransmitter secretion have been the subject of considerable investigation. Many of these investigations have centered on the cytoskeleton, which displays significant changes during growth cone elongation (Mitchison and Kirschner, 1988; Bentley and O'Connor, 1994; Letourneau, 1996). The removal, translocation, and addition of membrane also must be a part of the machinery of the growth cone. Although clearly important, these membrane dynamics have received considerably less attention, in part because of limitations of the methods available for studying living growth cones.

The pioneering work of Bunge first called attention to the existence of large membrane stores within growth cones (Bunge, 1973, 1977). A more contemporary picture of key aspects of growth cone membrane dynamics comes from investigations examining the formation and disappearance of large vacuoles (Dailey and Bridgman, 1993) and the movements of internal membranes (Dailey and Bridgman, 1989). Despite the considerable activity inferred from such studies the processes of membrane removal (endocytosis) and insertion (exocytosis) are still poorly understood in growth cones. A considerable literature from other

\footnotetext{
Received April 14, 1999; revised Aug. 6, 1999; accepted Aug. 13, 1999.

This work was supported by National Institutes of Health Grant NS24683. B.B. was supported by a postdoctoral fellowship from the Human Frontier Science Program. H.S. was supported by a postdoctoral fellowship from the Alexander von Humbolt Foundation. T.J.D. was supported in part via a postdoctoral fellowship from the Alberta Heritage Foundation for Medical Research. We acknowledge the excellent technical assistance of Ms. Kathleen Charters.

Correspondence should be addressed to Dr. S. B. Kater, Department of Neurobiology and Anatomy, University of Utah, Salt Lake City, UT 84132

Dr. Diefenbach's present address: Department of Physiology, Tufts University School of Medicine, 136 Harrison Avenue, Boston, MA 02111.

Dr. Stier's present address: University of Bonn, Institute of Anatomy and Cell Biology, Nussallee 10, 53115 Bonn, Germany.

Dr. Billup's present address: Department of Cell Physiology and Pharmacology, University of Leicester, Leicester LE1 9HN, UK.

Copyright (C) 1999 Society for Neuroscience 0270-6474/99/199436-09\$05.00/0
}

cell types suggests membrane events that might occur in growth cones (Mukherjee et al., 1997). For example, transcytosis [endocytosis of membrane from one site followed by reinsertion at another site (Hemar et al., 1997)] could, in principle, relocate membrane from one region of a growth cone to another (e.g., during turning). [The net addition of membrane during elongation, however, almost surely is derived from the distant cell body (Shea and Sapirstein, 1988; Popov et al., 1993; Craig et al., 1995; Dai and Sheetz, 1995) (see, however, Vance et al., 1991).] Alternatively, the retrograde transport of endosomes derived from the growth cone could convey information about the growth cone environment to the cell body and nucleus (Overly and Hollenbeck, 1996; Grimes et al., 1997; Zagrebelsky et al., 1998) (for review, see Oppenheim, 1996). Finally, growth cones can be stimulated to release neurotransmitter (Hume et al., 1983; Sun and Poo, 1987); this could involve synaptic vesicle exocytosis.

Recent technical advances that used the fluorescent membrane probe FM1-43 (Betz et al., 1996) have illuminated membrane dynamics in presynaptic terminals. Increased intracellular calcium evokes synaptic vesicle exocytosis, followed by the endocytotic retrieval of synaptic vesicle membrane. Endocytosis of FM143-stained membrane creates brightly fluorescent points within the terminal that have been identified as synaptic vesicles or vesicle clusters on physiological, immunocytochemical, and ultrastructural evidence (Betz and Bewick, 1992; Betz et al., 1992, 1996; Henkel et al., 1996).

The present investigation has taken advantage of the properties of FM1-43 to investigate membrane dynamics in the neuronal growth cone. This study was undertaken initially to explore a possible relationship between local membrane events deduced from earlier studies and growth cone neurotransmitter release. Our findings support such a relationship and go further: there appear to be two separable populations of endosomes (endocytotic vesicles) in the growth cone. Evoked endosomes form in 
response to depolarization and can be released by a subsequent depolarization. Constitutive endosomes, on the other hand, form even in the absence of depolarization and are not released by depolarization.

\section{MATERIALS AND METHODS}

Cell culture. Primary cultures were prepared from embryonic day 9-11 chick embryos as described previously (Schmidt and Kater, 1993, 1995; Schmidt et al., 1996). Ciliary ganglia were isolated in HBSS, treated for 15 min with a $2.5 \%$ trypsin solution (Life Technologies, Gaithersburg, $\mathrm{MD})$, rinsed in minimal essential medium (MEM; Life Technologies), and dissociated by trituration. Cells were plated on precleaned glass coverslips glued to holes in Falcon 1008 Petri dishes in MEM with 10\% fetal bovine serum (HyClone, Logan, UT), penicillin, and streptomycin (Life Technologies). The laminin-1 substrate $(2 \mu \mathrm{g} / \mathrm{cm}$; Collaborative Biomedical Research, Bedford, MA) was applied over poly-L-lysine for 1 $\mathrm{hr}$ before plating. After plating, the cultures were kept in a $37^{\circ} \mathrm{C}$ incubator with $5 \% \mathrm{CO}_{2}$ atmosphere. Cultures were prepared the same day of the experiments and were used 9-12 hr after plating, at which time numerous growth cones could be found that had not contacted other cells. Preplating was not required because non-neuronal cells were not abundant during this period. The survival factor bFGF was not used for these short-term cultures because neurons extended multiple neurites with large growth cones throughout the entire experimental period. $\alpha$-Latrotoxin was obtained from Alomone Laboratories (Jerusalem, Israel). FM1-43 was obtained from Molecular Probes (Eugene, OR).

FM1-43 staining. The principles underlying the process of staining endocytotic membrane with FM1-43 have been elucidated in synaptic vesicle recycling experiments in the presynaptic terminal (Betz and Bewick, 1992; Betz et al., 1996). FM1-43 is an amphiphilic compound that partitions, at equilibrium, between the lipid bilayer and the aqueous solution. FM1-43 is only weakly fluorescent in an aqueous environment but fluoresces brightly in membranes. Because the polar head group of FM1-43 is charged and cannot cross into a hydrophobic environment, the dye will not "flip" within the membrane. Therefore, when applied to the outside of a cell, FM1-43 remains in the outer leaflet of the plasma membrane, oriented away from the cytoplasm. If FM1-43 is in the plasma membrane during an endocytotic event, the endocytotic membrane will also contain FM1-43. The FM1-43 will be trapped on the inside of the endosome, still oriented away from the cytoplasm. This process of internalization of FM1-43-labeled membrane is known as loading.

After loading, the cells normally are washed with medium without FM1-43. The FM1-43 in the plasma membrane reequilibrates with the fresh aqueous medium, resulting in a loss of FM1-43 from the outside of the plasma membrane. The FM1-43 molecules in endosomes, however, remain trapped. The fluorescent endosomes are readily visible against the (now) unlabeled plasma membrane. Each fluorescent structure represents a product of endocytosis that formed only during the period of exposure to FM1-43. Because most endosomes are below the limit of resolution of the light microscope, fluorescent points could result from a single endosome or an aggregation of endosomes.

Fusion of a labeled endosome with the plasma membrane (exocytosis) results in the incorporation of endosomal membrane into the plasma membrane. FM1-43 is again exposed to the outside of the cell, and dye is rapidly lost as a result of reequilibration with the extracellular aqueous medium. This process is known as unloading. Fusion of endosomes with other, unlabeled intracellular organelles will not result in a net loss of fluorescence because the dye remains trapped by the lipid bilayer. In the presynaptic terminal a clear relationship exists among the destaining of FM1-43 on depolarization, fusion of vesicles with the plasma membrane, and the release of neurotransmitter (Betz et al., 1992; Betz and Bewick, 1993; Henkel et al., 1996).

For most experiments the cultures were loaded with FM1-43 and then fixed before fluorescence imaging to obtain large numbers of growth cones. FM1-43 stock solution was made up in purified water (Life Technologies, Grand Island, NY) to a concentration of $3 \mathrm{~mm}$. Aliquots of this solution were diluted in loading medium to a final concentration of $10 \mu \mathrm{M}$, vortexed, sonicated for $2 \mathrm{~min}$, centrifuged for $5 \mathrm{~min}$ at $7000 \times g$, and heated to $37^{\circ} \mathrm{C}$ before use. Two different types of loading medium were used. Depolarizing loading medium consisted of MEM mixed with isotonic $\mathrm{KCl}$ to produce a final $\mathrm{KCl}$ concentration of $60 \mathrm{~mm}$. Normal loading medium was MEM. The loading medium was added to the culture dishes under low light conditions for the predetermined loading period. Cultures then were rinsed rapidly five times with $2 \mathrm{ml}$ of $37^{\circ} \mathrm{C}$ MEM (without fetal bovine serum) over a course of $1 \mathrm{~min}$. This rinse period ensured a complete washout of excess FM1-43 from the culture and from external membranes. Cultures were rinsed twice with ice-cold $4 \%$ paraformaldehyde with $0.12 \mathrm{M}$ sucrose and then fixed with $4 \%$ paraformaldehyde for $15 \mathrm{~min}$ at $4^{\circ} \mathrm{C}$ in the dark. After fixation the cultures were rinsed three times with ice-cold $10 \mathrm{~mm}$ PBS and kept in PBS at $4^{\circ} \mathrm{C}$ in the dark until imaging. Loading followed by immediate fixation resulted in very high levels of background staining, which made the identification of endosomes problematic. Cultures treated with FM1-43 that were fixed and kept at $4^{\circ} \mathrm{C}$ could be imaged the next day without a noticeable loss of signal. For experiments involving the observation of living growth cones, the cultures were loaded with FM1-43 and rinsed with MEM without subsequent fixation.

Living growth cones also were loaded individually by micropipette (tip diameter, $\sim 20 \mu \mathrm{m})$ application of the loading medium $(20-40 \mu \mathrm{M}$ FM1-43). The distance of the pipettes from the growth cones was $\sim 75$ $\mu \mathrm{m}$. One series of experiments used a dual micropipette apparatus to restrict the application of FM1-43 more precisely. Two micropipettes were positioned in parallel with the tips adjacent to each other, $\sim 20 \mu \mathrm{m}$ apart. One micropipette was filled with loading medium and the other with MEM alone. Positive pressure was applied to both pipettes simultaneously. The two streams met and formed a stable boundary, resulting in a precise restriction of FM1-43 labeling. Cultures were positioned so that the boundary of FM1-43 labeling was within the growth cone; therefore, FM1-43 would label only endosomes created from that labeled membrane, i.e., from growth cone membrane. Given the minute volumes of FM1-43 pulsed onto growth cones, rapid dilution of the dye in the surrounding medium makes it difficult to determine the final FM1-43 concentration applied at the membrane.

Imaging and quantitative analysis of FM1-43 staining. FM1-43-loaded growth cones were imaged by using an inverted epifluorescence microscope (Diaphot 300, Nikon) with $60 \times$ (PlanApo $60 \times / 1.4$ numerical aperture; Nikon) or $100 \times$ (phase $100 \times / 1.4$ numerical aperture; Zeiss) oil immersion objectives. The resolution limit is $\sim 220 \mathrm{~nm}$ as determined from subresolution $(57 \mathrm{~nm})$ fluorescent beads. Illumination was provided by a $100 \mathrm{~W}$ mercury lamp through $1 \mathrm{~mm}$ fiber optic. A computercontrolled shutter minimized illumination of the preparation. FM1-43 fluorescence images were acquired by using fluorescein optics $(480 \mathrm{~nm}$ excitation, $505 \mathrm{~nm}$ dichroic mirror, and $520 \mathrm{~nm}$ emission wavelengths) with a cooled CCD camera (Photometrics, Tucson, AZ) and IPLab Spectrum image acquisition software (Scanalytics, Fairfield, VA) on a Power Macintosh. A computer-controlled microscope stage was used for acquiring vertical series of images through the entire depth of a growth cone at $0.25 \mu \mathrm{m}$ increments and for relocating specific growth cones when needed. In all cases, only growth cones that had not contacted other cells were included in our analysis. Living cultures were maintained at $37^{\circ} \mathrm{C}$ in a $5 \% \mathrm{CO}_{2}$ atmosphere on the microscope stage.

Image series were analyzed with IPLab Spectrum software. For some experiments the labeled endosomes were counted, with the number being normalized to the surface area of the growth cone. The heavily labeled growth cones resulting from evoked endocytosis, however, increased the probability that multiple endosomes would be clustered into an individual fluorescent point. Therefore, for the majority of experiments the intensity of FM1-43 fluorescence in a growth cone was obtained by choosing the image plane with the greatest number of stained endosomes, measuring the average fluorescence intensity across the entire growth cone area, and subtracting the nonspecific background fluorescence. Background fluorescence was determined from the average of three regions in the growth cone between stained endosomes. Image acquisition parameters were kept fixed for all of the experiments; therefore, intensity values can be compared between growth cones and between experiments. All intensity values are presented in arbitrary units. The time course of constitutive endocytosis was quantified by integrating the total fluorescence of the identified endosomes and subtracting the autofluorescence of the growth cone. This approach, although slightly more sensitive, was considerably more time-consuming and was difficult to implement in heavily labeled growth cones. It therefore was used only for the constitutive-loading time course experiment (Fig. 3).

Statistical analysis used Student's two-way $t$ test, unpaired, with a $95 \%$ confidence interval. Statistical analysis of the results was facilitated by the large numbers of labeled growth cones available for analysis in each culture. All values are expressed as the mean \pm SEM. 

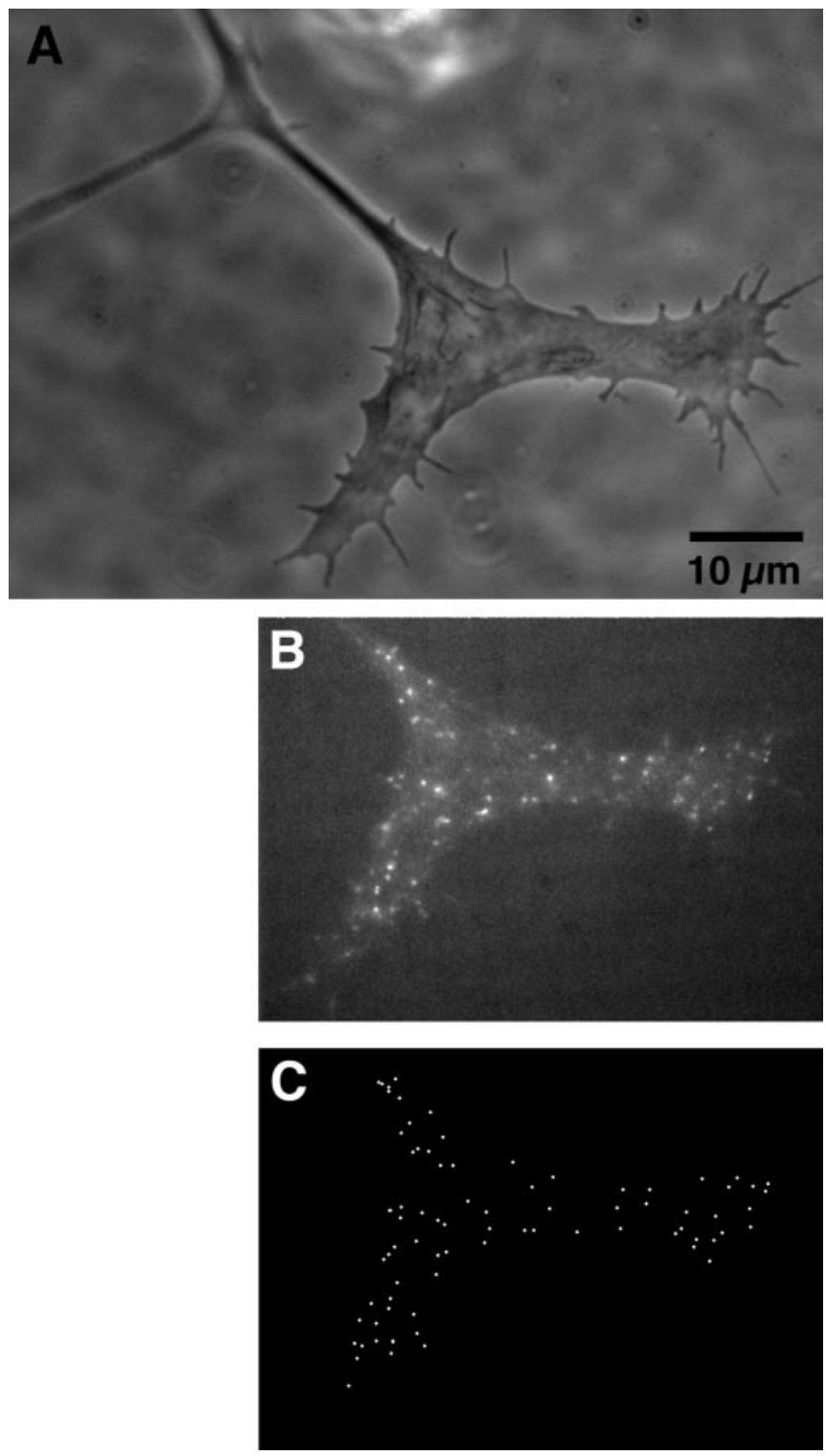

Figure 1. FM1-43-labeled endosomes in ciliary ganglion neuronal growth cones. $A$, Phase-contrast image of a living growth cone. $B$, Fluorescence image of a growth cone $30 \mathrm{sec}$ after a $20 \mathrm{sec}$ application of depolarizing medium containing FM1-43 $(40 \mu \mathrm{M})$ from a micropipette. Numerous small intensely stained endosomes can be distinguished individually. Note the absence of endosomes in filopodia. $C$, A dot map of the same growth cone showing the distribution of individually labeled endosomes in the growth cone.

\section{RESULTS}

\section{FM1-43 labeling of endosomes in growth cones}

Chick ciliary neuronal growth cones internalized fluorescence when they were exposed to depolarizing medium $(60 \mathrm{~mm} \mathrm{KCl})$ containing FM1-43 (10 $\mu \mathrm{M})$ (Fig. 1). The fluorescent label initially was restricted to numerous small fluorescent endosomes or endosome clusters. There was no obvious pattern of endosome distribution in the growth cone; generally, endosomes were dispersed evenly throughout the body of the growth cone, along the neurite, and in the soma, although endosomes rarely were observed within filopodia. Endosomes were highly mobile and displayed rapid movements both within the growth cone and from the growth cone to the adjacent neurite. With longer intervals between loading and fixation, larger labeled structures often were observed, although whether this represents true membrane fusion of endosomes into larger structures or an aggregation of endosomes is not clear. Larger particles occasionally were observed to separate into smaller ones. Occasionally, endosomes suddenly disappeared in an "exocytosis-like" event (see below).

The total amount of fluorescence seen in growth cones increased with increasing loading time although, within a single growth cone, different individual fluorescent points could have different fluorescence intensities. Growth cones loaded for $>60$ sec often were so bright that endosomes no longer could be distinguished as individual fluorescent points. Shorter loading periods, followed quickly by thorough washing, reduced the complexity of the images. Growth cones remained motile throughout these experiments. Loading at $4^{\circ} \mathrm{C}$ resulted in no internalized fluorescence, suggesting that an energy-dependent process (e.g., endocytosis) is involved in the FM1-43 internalization.

The growth cone appeared to provide higher resolution images than the original preparation described by Betz (Betz and Bewick, 1992). This is likely attributable to the differences in preparations, with the large flattened growth cone being especially favorable for observation. In our preparations the unit fluorescent particle is at the level of resolution $(\sim 220 \mathrm{~nm})$. If we interpret our fluorescent points as vesicles or clusters of vesicles [as did Betz et al. (1992); Ryan et al. (1993); Henkel et al. (1996); Murthy et al. (1997)], a single fluorescent point could represent a single vesicle or a square cluster of as many as four $100 \mathrm{~nm}$ vesicles; clustering could account for the variation in intensity of different fluorescent points. In addition, individual endosomes, which were generated at different times during the brief FM1-43 labeling period (while FM1-43 was still equilibrating into the plasma membrane), easily could have very different membrane concentrations of FM1-43.

\section{Cobalt blocks evoked endocytosis}

Although the endocytotic portion of the synaptic vesicle recycling system appears to be, in large part, calcium-independent (Ryan et al., 1993; Wu and Betz, 1996), it is clear that calcium regulates exocytosis and that exocytosis is the proximate trigger for endocytosis at the presynaptic terminal (Augustine et al., 1996; Lledo, 1997). In the presynaptic terminal, therefore, calcium influx normally is required during stimulation to obtain FM1-43 loading. We tested for a calcium dependence of depolarization-evoked endocytosis by including the general calcium channel blocker $\mathrm{CoCl}_{2}$ (2 mM) with FM1-43 in the depolarizing medium. Cultures were incubated with FM1-43-containing medium, rapidly washed, and then fixed.

Figure $2 A$ illustrates that the coapplication of cobalt with FM1-43 resulted in a dramatic reduction in evoked endosomes in growth cones. Figure $2 B$ shows the results of a quantitative analysis of several such experiments. Blocking calcium influx resulted in a significant reduction in FM1-43 labeling $(\mathrm{KCl}$ loading, $89.7 \pm 12.6, n=61$ vs $\mathrm{KCl}$ loading in $\mathrm{CoCl}_{2}, 8.52 \pm 4.23$, $n=25 ; p<0.0001)$. A comparison of these results with the literature from the presynaptic terminal revealed a significant difference, however. In the presynaptic terminal there was virtually no FM1-43 labeling in the absence of a calcium influx; in the neuronal growth cone, however, there was still significant endocytosis. This residual endocytosis may represent a distinct pathway that is independent of depolarization. Given the characteristics described below, we refer to this pathway as "constitutive" endocytosis, recognizing that, eventually, unique regulators also might be identified. 

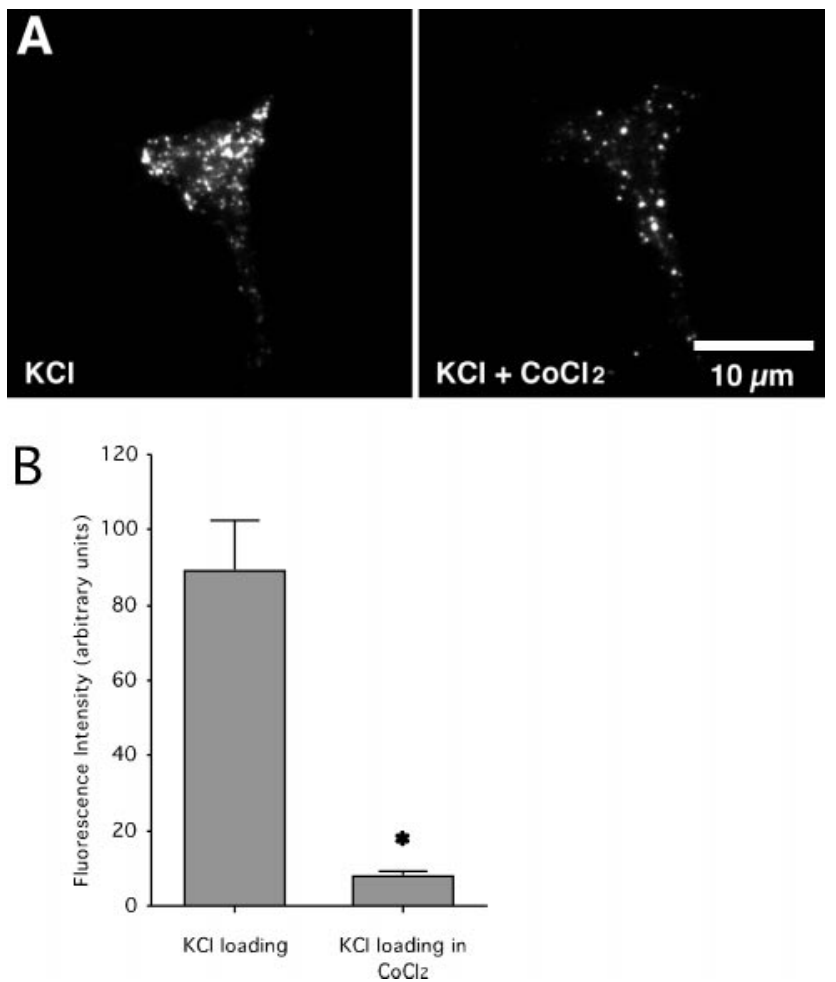

Figure 2. Evoked loading of FM1-43 is blocked by cobalt. A, The left panel shows a fixed growth cone that had been loaded for $10 \mathrm{sec}$ with depolarizing medium containing FM1-43 $(10 \mu \mathrm{M})$. The right panel shows the effect of adding $\mathrm{CoCl}_{2}(2 \mathrm{~mm})$ to the loading medium. Cobalt cotreatment resulted in less endocytosis, as evidenced by fewer endosomes. B, Depolarization of growth cones in the presence of cobalt resulted in a $85 \%$ reduction in average growth cone fluorescence when compared with growth cones that were depolarized without cobalt. The number of growth cones/experiments: $\mathrm{KCl}$ loading, 61/6; $\mathrm{KCl}$ loading in $\mathrm{CoCl}_{2}, 25 / 3 .{ }^{*} p<0.0001$, Student's $t$ test.

\section{Constitutive (depolarization-independent) endocytosis occurs in the growth cone}

To characterize constitutive endocytosis, we exposed growth cones to FM1-43 in normal culture medium (that is, without a depolarizing agent). As with evoked endocytosis, growth cones maintained normal motility throughout the experiments. Also, there was no obvious pattern to endosome distribution, and endosomes were highly mobile and appeared to condense into larger structures over time. Growth cones showed increased staining with time (Fig. $3 A$ ). The rate of endocytosis was measured by integrating the total FM1-43 fluorescence throughout the entire growth cone (except filopodia). A linear relationship between exposure time and integrated FM1-43 fluorescence intensity was obtained (Fig. $3 B$ ). In contrast to evoked endocytosis, $\mathrm{CoCl}_{2}$ (2 mM) did not affect constitutive endocytosis (control loading, $12.8 \pm 1.5, n=31$; loading in $\left.\mathrm{CoCl}_{2}, 15.8 \pm 2.0, n=27 ; p>0.05\right)$.

The rates of evoked and constitutive endocytosis can be compared directly by using data from the $10 \mathrm{sec}$ loading experiments. Evoked endocytosis resulted in an average of $0.58 \pm 0.06$ endosomes $/ \mu \mathrm{m}^{2}$ of growth cone membrane during the $10 \mathrm{sec}$ loading period. Constitutive endocytosis occurred at a slower rate: $0.19 \pm$ 0.06 endosomes $/ \mu \mathrm{m}^{2}$ for constitutive endocytosis. Even with a lower activity, however, the average constitutive endocytotic activity would result in a complete turnover of growth cone membrane within $60 \mathrm{~min}$, assuming $100 \mathrm{~nm}$ diameter endosomes. This, of course, is likely to be an underestimate of the actual rate of
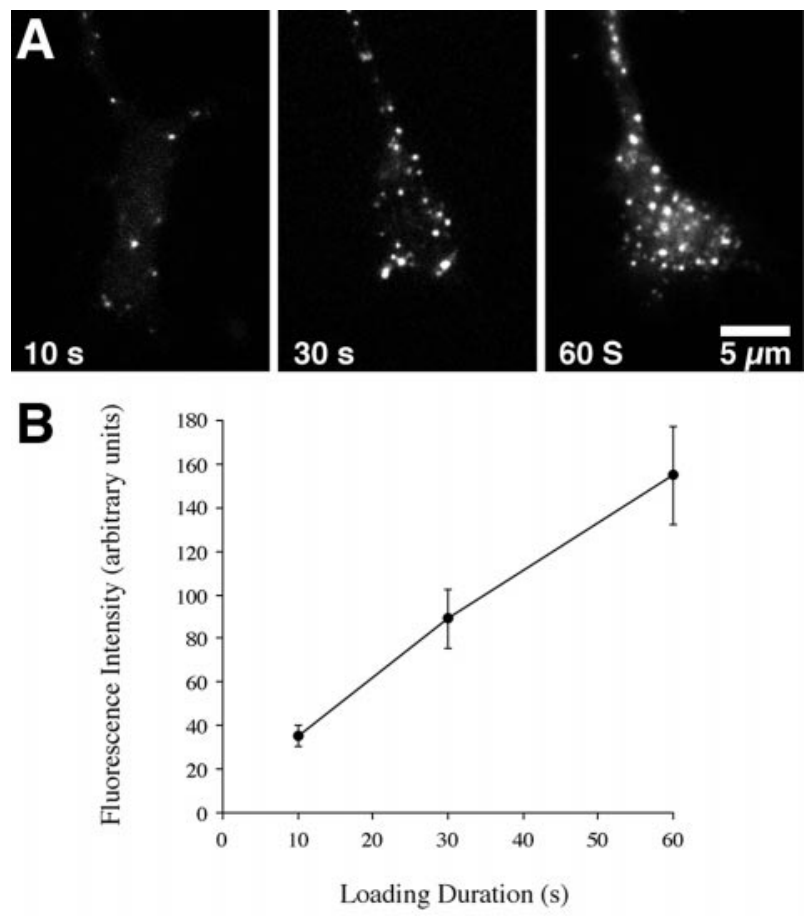

Figure 3. Constitutive endocytosis in growth cones. A, Growth cones loaded with FM1-43 in normal medium show constitutive endocytotic activity that does not require depolarization and is not blocked by cobalt. Shown are three growth cones that were loaded with FM1-43 for 10, 30, or $60 \mathrm{sec}$ before fixation. $B$, The integrated fluorescence of the endosomes increased in proportion to the loading time. The number of growth cones/experiments: for $10 \mathrm{sec}, 35 / 6$; for $30 \mathrm{sec}, 24 / 5$; for $60 \mathrm{sec}, 52 / 10$.

membrane turnover if multiple vesicles have aggregated into a single fluorescent point. In fact, constitutive endocytosis was observed at rates that would result in the equivalent of a complete turnover of the growth cone in $<30 \mathrm{~min}$.

\section{Endocytosis occurs locally within the growth cone}

The endosomes visible in labeled growth cones could be derived from endocytosis in the growth cone proper or from endocytosis in neurites with subsequent shipment to the growth cone during the wash period. Although it is unlikely that the observed high levels of endocytosis could be attributable to such a mechanism, this possibility was tested directly. Figure $4 A$ shows the location of a growth cone. Two pipettes were positioned adjacent to each other so that their output streams were adjacent, creating a sharp boundary of FM1-43 labeling and restricting the labeling to the distal end of the growth cone, as seen in the intense staining of only that region (Fig. $4 B$ ). Then both pipettes were removed, and the growth cone plasma membrane was allowed to de-stain in normal medium. When imaged $30 \mathrm{sec}$ later, endosomes were observed, but they were restricted to the distal end of the growth cone (Fig. $4 C, D$ ), the region of the growth cone that previously had shown intense fluorescence in the plasma membrane. Similar results were obtained in each of four growth cones and in each of six growth cones loaded with FM1-43 in depolarizing medium. Thus, both constitutive and evoked endosomes can be derived from growth cone plasma membrane.

Although this study was concerned primarily with the process of endocytosis, the experiments did allow some notable observations on the subsequent movements of labeled endosomes. Two important changes in endosome distribution have been observed: (1) endosomes formed in one region of the growth cone are 

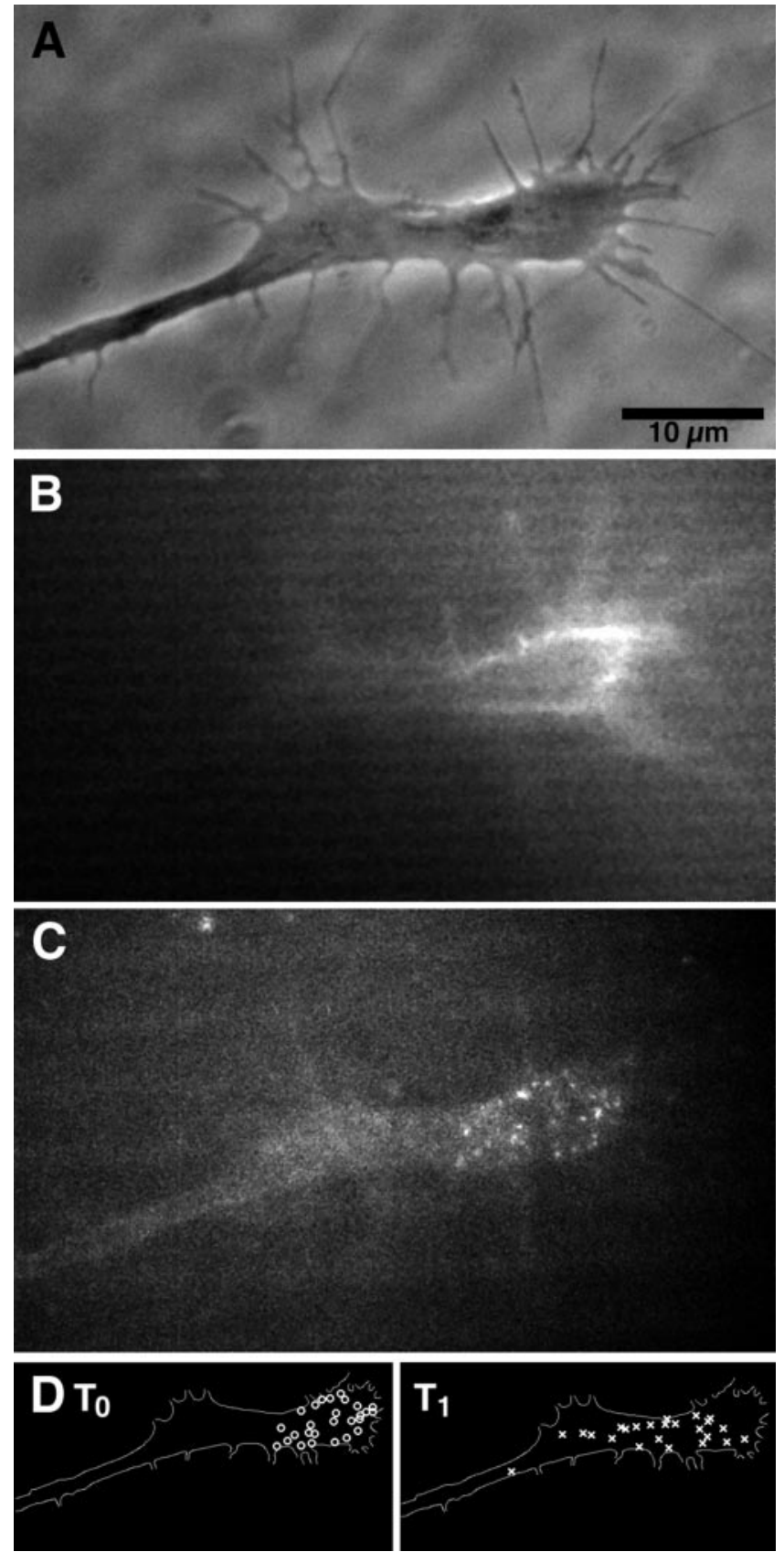

Figure 4. Endocytosis of growth cone membrane and subsequent redistribution of labeled endosomes. $A$, Phase-contrast image of a living growth cone before FM1-43 application. B, The same growth cone viewed with fluorescence optics during the local FM1-43 application from a micropipette. FM1-43 was restricted to the distal half of the growth cone by a second micropipette that applied normal medium without the dye (see Materials and Methods). During FM1-43 application the exposed plasma membrane of the growth cone became intensely fluorescent. Note that filopodia also were stained intensely during the loading process. Because of the restriction in the dye-containing medium stream, only the distal half of the growth cone plasma membrane was exposed to FM1-43. $C$, Numerous distinct fluorescent endosomes imaged $30 \mathrm{sec}$ after local application of FM1-43. The endosomes appeared only in the region in which the growth cone membrane had been exposed to FM1-43. D, Left $\left(T_{0}\right)$, The stained endosomes identified in $C$ were each marked by a white circle to depict their distribution. D, Right $\left(T_{0}\right)$, The same growth cone, imaged 7 min later, shows a redistribution of endosomes away from their place of origin to more proximal regions of the growth cone and into the neurite. Each endosome was marked by a white $X$. The boundary of the growth cone is marked in white. generally mobile and will redistribute throughout the growth cone and into proximal neurites, and (2) endosomes can disappear via a process that almost certainly represents exocytosis.

Endosomes formed in one region of the growth cone redistributed throughout the growth cone and into proximal neurites, as illustrated by Figure $4 D$. The left panel $\left(T_{0}\right)$ shows the distribution of endosomes within $30 \mathrm{sec}$ of FM1-43 labeling; the right panel $\left(T_{1}\right)$ shows the distribution of the same endosomes $7 \mathrm{~min}$ later. It is clear that FM1-43-stained endosomes are not confined to the initial site of membrane uptake but, rather, undergo excursions throughout the growth cone and into the neurite. It should be pointed out that many different types and magnitudes of movement are observed. Some endosomes appear to move randomly within a small area, whereas others appear to move purposefully in one direction. In fact, not all endosomes show detectable movement; some endosomes, especially the larger ones, have remained at a fixed position in the growth cone for up to 30 $\min$.

Occasionally, endosomes suddenly disappeared in an "exocytosis-like" event. In principle, one would expect to be able to observe exocytosis directly, although a single FM1-43 labeled endosome is below the limits of resolution of the fluorescence microscope. On the fusion of the endosome with the plasma membrane there should be a sudden decrease in fluorescence intensity as the FM1-43 diff uses both laterally through the membrane away from the site of exocytosis and away from the membrane into the medium (Henkel et al., 1996); this process is the basis of the stimulus-evoked unloading described in the presynaptic terminal. A similar process was observed in the growth cone (Fig. 5). At the start of this time sequence two brightly labeled evoked endosomes were visible at the tip of the growth cone. Although both endosomes were stable for many seconds, one of the endosomes (arrow) underwent a rapid and irreversible loss of fluorescence starting at the $6 \mathrm{sec}$ time point. The second endosome, however (arrowhead), retained both its position and its fluorescence during the same period. The graph in Figure 5 shows a plot of normalized fluorescence intensity $\left(F / F_{\mathrm{o}}\right)$ as a function of time for the two endosomes. Whereas the endosome that remained visible showed no appreciable change in the fluorescence intensity over time (dotted line), the endosome that disappeared had an intensity profile that dropped to near zero at the time of disappearance (solid line) and remained at background levels for the remainder of the observation period (>30 sec). This is virtually identical to the process observed in the recent literature on synaptic transmission. Although these spontaneous exocytotic events were rare, they nevertheless suggest that membrane retrieved via evoked endocytosis from the growth cone can be reincorporated into the plasma membrane pool of the growth cone.

\section{Stimulated exocytosis of evoked endosomes}

Studies of the presynaptic terminal have shown that labeled synaptic vesicles can be released via exocytosis after stimulation of the terminal. A similar evoked exocytosis of evoked endosomes also was seen in the growth cone by using four different approaches.

Two series of experiments used imaging of living growth cones. In the first, individual growth cones were labeled by local micropipette application of FM1-43 $(20 \mu \mathrm{M})$ in depolarizing medium (Fig. $6 A_{1}$ ). After a 2 min wash period a second pipette containing depolarizing medium without FM1-43 was used to depolarize the labeled growth cone. This depolarization resulted in the 

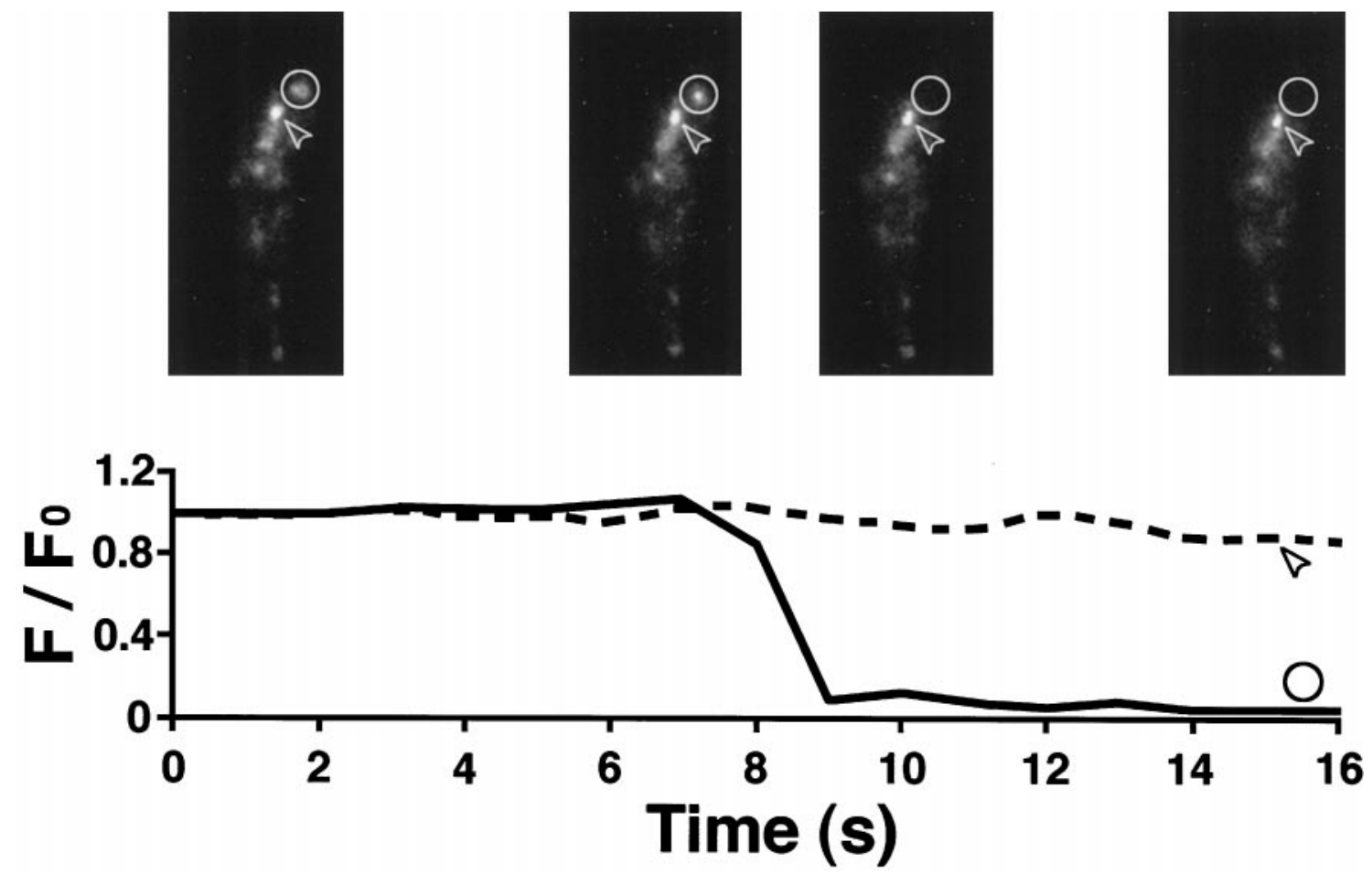

Figure 5. Spontaneous disappearance of endosomes from growth cones. This time series shows an example of an endosome (in the circle) that, after an extended period of immobility, disappeared within a $2 \mathrm{sec}$ period (third panel). A second endosome (arrowhead) remained stationary and within the same focal plane during the entire period. The graph shows the normalized fluorescence intensity $\left(F / F_{\mathrm{o}}\right)$ for the endosome that disappeared $($ solid line) and the endosome that remained (dotted line).
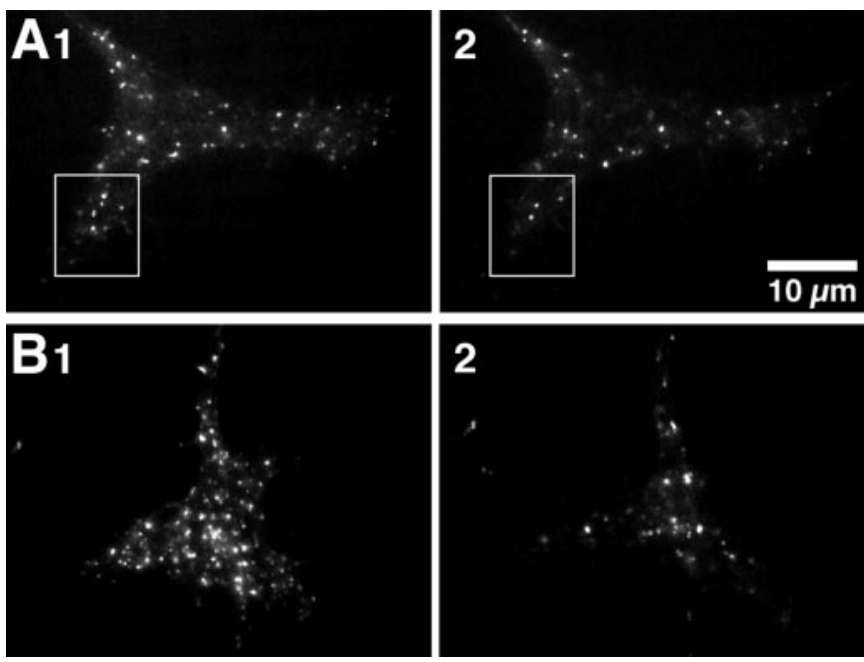

Figure 6. Evoked endosomes are released by subsequent depolarization. $A$, A growth cone was loaded by micropipette application of depolarizing medium containing FM1-43 $(40 \mu \mathrm{M})\left(A_{1}\right)$. Then 2 min later the growth cone was depolarized a second time without FM1-43 $\left(\mathrm{A}_{2}\right)$. Depolarization resulted in a reduction in the number of visible endosomes, as emphasized by the region outlined by the white rectangle. $B$, A growth cone in a culture that was loaded with depolarizing medium for $10 \mathrm{sec}$ before imaging $\left(B_{1}\right)$. Then 2 min later the entire culture was depolarized again by the addition of a depolarizing medium without FM1-43 $\left(\mathrm{B}_{2}\right)$. Depolarization resulted in a dramatic reduction in the number of endosomes.

exocytosis of FM1-43-labeled endosomes; although some endosomes persisted, many were clearly absent after depolarization (Fig. $6 A_{2}$ ). In a second series of experiments the locations of three to five growth cones were stored by using the computer- controlled microscope stage. Then the cultures were loaded by bath application of a depolarizing medium containing FM1-43 $(10 \mu \mathrm{M})$ and washed. The microscope stage was moved rapidly to the locations of the selected growth cones, and they were imaged. After a 2 min wash period the cultures were depolarized again for $10 \mathrm{sec}$ with $\mathrm{KCl}(60 \mathrm{~mm})$ without FM1-43. The growth cones were relocated and reimaged. This approach also demonstrated a clear reduction in the number of endosomes after depolarization (Fig. 6B).

For a third approach the growth cones were loaded with FM1-43 $(10 \mu \mathrm{M})$ in depolarizing medium $(60 \mathrm{~mm} \mathrm{KCl})$, rinsed for $2 \mathrm{~min}$, depolarized a second time for $10 \mathrm{sec}$, and immediately fixed. In this way many growth cones could be imaged in each culture dish without concern for time-dependent changes in staining quality or localization. Growth cones exposed to a second depolarization had one-half of the total FM1-43 fluorescence (KCl-evoked unloading, $43.2 \pm 7.2, n=66$ ) seen in control growth cones (which did not receive a second depolarization) (control, $89.7 \pm 12.6, n=61 ; p<0.005$; Fig. 7). Thus, on a population level, depolarization resulted in a significant reduction in the fluorescence contributed by evoked endosomes. A $10 \mathrm{sec}$, $60 \mathrm{~mm} \mathrm{NaCl}$ pulse did not result in the exocytosis of evoked endosomes $(89.6 \pm 20.8, n=37 ; p>0.05$ as compared with control growth cones).

The fourth approach used $\alpha$-latrotoxin (black widow spider venom) as a general secretagogue. $\alpha$-Latrotoxin evokes massive neurotransmitter release from presynaptic terminals without depolarization (Matteoli et al., 1988; Henkel and Betz, 1995). $\alpha$-Latrotoxin ( $3 \mathrm{nM}$ ) was even more effective in causing exocytosis than a $10 \mathrm{sec}$ depolarization (latrotoxin-evoked unloading, $19.9 \pm$ 4.3, $n=30$; Fig. 7). 

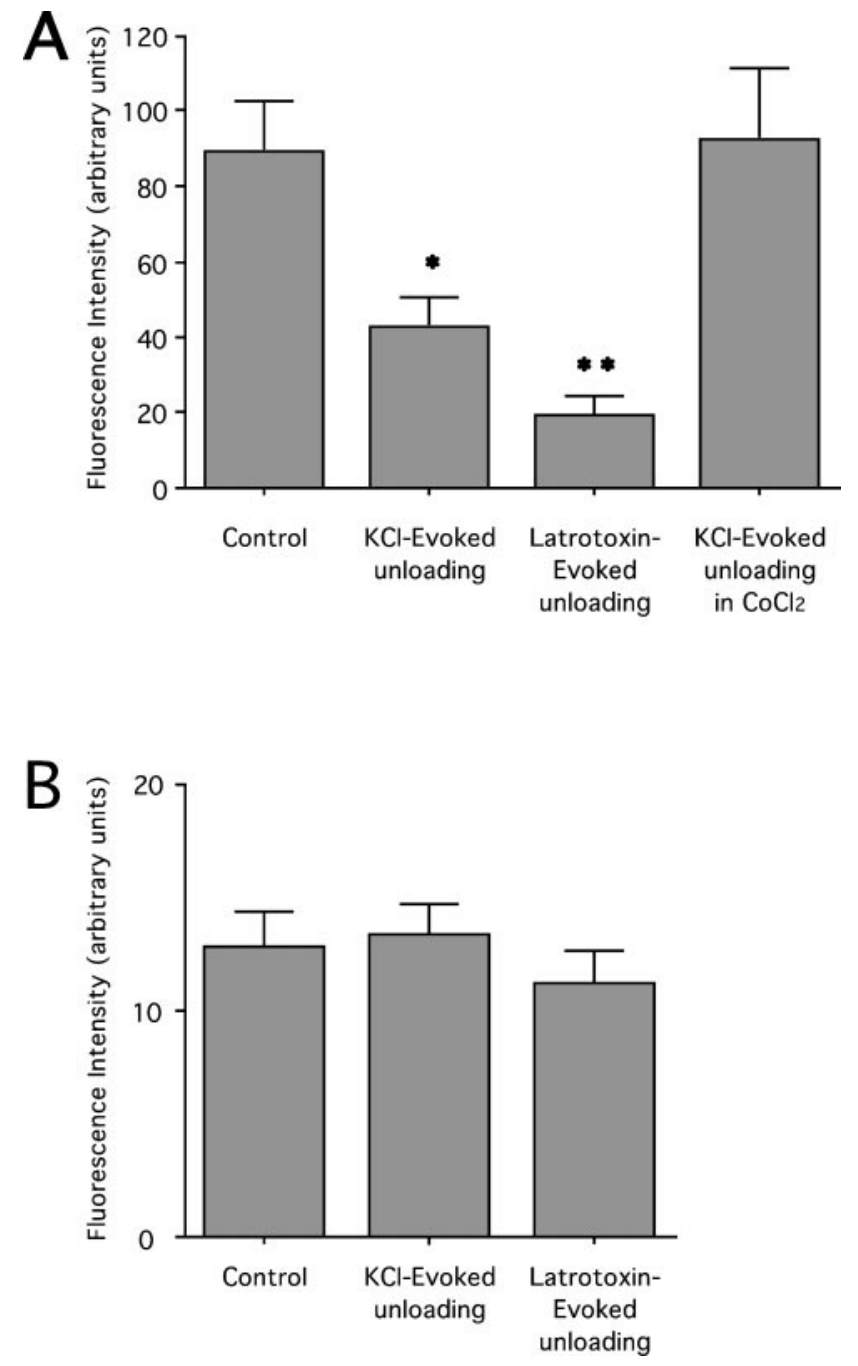

Figure 7. Differential susceptibility of evoked and constitutive pools to unloading by depolarization and latrotoxin. $A$, Evoked endosomes can be released by depolarization and latrotoxin. Growth cones were loaded with depolarizing medium, rinsed for $2 \mathrm{~min}$, and fixed (Control). A second depolarization ( $\mathrm{KCl}$-Evoked unloading) resulted in a $>50 \%$ reduction in average fluorescence intensity. The addition of latrotoxin $(3 \mathrm{nM})$ resulted in an even greater reduction (Latrotoxin-Evoked unloading). The depolarization-evoked endosome release was blocked by $\mathrm{CoCl}_{2}(\mathrm{KCl}$ Evoked unloading in $\mathrm{CoCl}_{2}$ ). The number of growth cones/experiments: control, 61/6; KCl-evoked unloading, 66/5; latrotoxin-evoked unloading, 30/3; KCl-evoked unloading in $\mathrm{CoCl}_{2}, 30 / 3 .{ }^{*} p<0.01$; * $p<0.001 . B$, Constitutively loaded endosomes are unaffected by depolarization and latrotoxin. Growth cones were loaded in a nondepolarizing medium for $30 \mathrm{sec}$ and rinsed. Labeled growth cones then either were fixed directly (Control) or exposed to a depolarizing medium (KCl-Evoked unloading) or to latrotoxin (3 nM; Latrotoxin-Evoked unloading), followed by fixation. Neither treatment caused a significant reduction in FM1-43 fluorescence in growth cones as compared with control growth cones. The number of growth cones/experiments: control, 31/3; KCl-evoked unloading: 29/3; latrotoxin-evoked unloading, 21/3.

Exocytosis in the presynaptic terminal generally requires a significant calcium influx. We tested whether the depolarizationevoked exocytosis of evoked endosomes was blocked by extracellular cobalt. Growth cones loaded with FM1-43 under depolarizing conditions were depolarized for a second time with depolarizing medium containing $\mathrm{CoCl}_{2}(2 \mathrm{~mm})$. There was no measurable exocytosis of evoked endosomes in the presence of $\mathrm{CoCl}_{2}\left(\mathrm{KCl}\right.$-evoked unloading in $\left.\mathrm{CoCl}_{2}, 92.9 \pm 19, n=47\right)$ as compared with control growth cones that had not been subjected to a second depolarization.

\section{No stimulated exocytosis of constitutive endosomes}

The constitutive endocytotic pathway was distinct from the evoked endocytotic pathway in its dependence on depolarization. A final series of experiments tested whether constitutive endosomes also could be released by depolarization. Growth cones were loaded under nondepolarizing conditions with FM1-43 for a $30 \mathrm{sec}$ period, rinsed, depolarized with $60 \mathrm{~mm} \mathrm{KCl}$ for $10 \mathrm{sec}$, and immediately fixed. Depolarization did not reduce the fluorescence of constitutively labeled growth cones (control, $12.8 \pm$ 1.5, $n=31$; KCl-evoked unloading, $13.3 \pm 1.4, n=29 ; p>0.8$ ) (Fig. 7). Treatment with $\alpha$-latrotoxin ( $3 \mathrm{~nm}$ ) also did not release constitutive endosomes (latrotoxin-evoked unloading, $11.2 \pm 1.4$, $n=21 ; p>0.4$ vs control). The failure of constitutive endosomes to be released on depolarization was confirmed by direct counts of endosomes within growth cones. Constitutive endocytosis resulted in $0.19 \pm 0.01$ endosomes $/ \mu \mathrm{m}^{2}$ membrane surface area. Growth cones loaded constitutively and subsequently depolarized had $0.17 \pm 0.1$ endosomes $/ \mu \mathrm{m}^{2}$ ( $p>0.4$ vs control). Growth cones treated with latrotoxin had $0.19 \pm 0.1$ endosomes $/ \mu \mathrm{m}^{2}$ ( $p>0.7$ vs control). The results from these experiments, irrespective of mode of analysis, further distinguish between evoked endosomes and constitutive endosomes.

\section{DISCUSSION}

Surprisingly high levels of ongoing endocytotic activity were found in growth cones. Furthermore, endocytotic activity was observed throughout the growth cone, suggesting that all growth cone membrane is competent with regard to this activity. Perhaps the most notable finding from the present investigation is that endocytosis occurs via two pathways that are separable both in terms of their stimulus dependence and in terms of the subsequent fate of the endosomes. Evoked endocytosis is similar to the synaptic vesicle recycling observed in presynaptic terminals. Constitutive endocytosis, however, is distinct and could well be subserving growth cone-specific functions because it has not been reported in studies of the presynaptic terminal.

\section{Membrane dynamics in the neuronal growth cone}

Membrane dynamics have been studied extensively in numerous cell types. Within epithelial cells, transcytosis provides a means of relocating membrane lipids and proteins to different regions of the cell as well as transporting extracellular substances from one side of the cell to another. Within neurons the processes of anterograde and retrograde vesicle transport may serve a related function over long distances or, perhaps, over much shorter distances such as between portions of a single growth cone. In the nerve terminal a specialized process coupling endocytosis to exocytosis underlies synaptic vesicle recycling; after calciumdependent exocytosis the synaptic vesicles are pinched off from the plasma membrane via endocytosis and refilled with transmitter to become available for subsequent use. This basic description, now based on $>40$ publications that used FM1-43, provides a substantial foundation and good point of comparison for parallel experiments on the neuronal growth cone.

Endocytosis appears to be required for outgrowth (Kim and Wu, 1987; Masur et al., 1990; Torre et al., 1994; Mundigl et al., 1998). The recent literature contains, however, few investigations that focus primarily on the neuronal growth cone. Numerous vesicle populations have been found in ultrastructural studies of 
Table 1. Characterization of the two endocytotic pools observed in growth cones

Evoked endocytotic pool Constitutive endocytotic pool

Endocytosis evoked by depolarization

Formation of endosomes blocked by cobalt

Endosomes released by depolarization

Endosomes released by $\alpha$-latrotoxin

Endosome release blocked by cobalt

$\begin{array}{lc}+ & - \\ + & - \\ + & - \\ + & - \\ + & \text { NA }\end{array}$

growth cones; the complexity of the advancing growth cone, however, makes it difficult to ascribe specific identities to these vesicles absolutely. Dailey and Bridgman paint a picture of dynamic endoplasmic reticulum (Dailey and Bridgman, 1989) and large vacuoles (Dailey and Bridgman, 1993) within growth cones. Although these structures are clearly larger than the endosomes observed with FM1-43, their electron micrographs show vesicles of smaller dimensions that would fit those observed in the present study. It is interesting to note that the large membrane disks observed by Cheng and Reese (1987) were not observed in our normal experiments. Longer intervals between loading and observation might be needed for endosomes to fuse with and become integrated into these disks (Wessells et al., 1974).

\section{Comparison of membrane dynamics between the growth cone and the presynaptic terminal}

There are both similarities and differences between the growth cone and the presynaptic terminal. Notably, both display significant evoked endocytosis. At the presynaptic terminal this endocytosis occurs as a direct result of the exocytotic activity resulting in neurotransmitter release (Betz and Bewick, 1992, 1993), with the apparent calcium dependence attributable to calciumdependent exocytosis and not a direct calcium dependence of endocytosis (Ryan et al., 1993; Wu and Betz, 1996) [see, however, von Gersdorff and Matthews, 1994]. Growth cones also can be stimulated to release neurotransmitters (Hume et al., 1983; Sun and Poo, 1987). The evoked endocytotic pool we have observed likely represents synaptic vesicles and results from the depolarization-evoked exocytotic release of neurotransmitter from the growth cone. This conclusion is based on the facts that (1) these endosomes form in response to stimulation (depolarization); (2) extracellular cobalt blocks this endocytosis, suggesting a requirement for calcium influx (although blocking calcium influx blocks evoked endocytosis, it is not clear whether calcium is acting directly or indirectly, e.g., by stimulating exocytosis); (3) depolarization releases these endosomes in a calcium-dependent manner; and (4) $\alpha$-latrotoxin releases this pool. Furthermore, several laboratories have demonstrated, as part of the studies of synaptogenesis (Kraszewski et al., 1995; Dai and Peng, 1996), that synaptic vesicle recycling occurs in the elongating axon.

In contrast to the presynaptic terminal, the growth cone also displays significant constitutive endocytosis. Extracellular cobalt does not block formation of the constitutive pool, suggesting both that constitutive endocytosis does not require exocytotic activity and that constitutive endocytosis is calcium-independent. Furthermore, this pool is not released by depolarization or by $\alpha$-latrotoxin. Thus, there appear to be two distinct endocytotic pools in the growth cone that are under separate control mechanisms (see Table 1). Vesicles derived from each pathway appear to be constrained to different possible fates.

Although several studies (Pieribone et al., 1995; Kuromi and Kidokoro, 1998, 1999) have demonstrated separable synaptic vesicle pools in presynaptic terminals (a readily releasable pool and a reserve pool), the constitutive pool is likely to be yet a different pool. The two synaptic vesicle pools are generated by the same depolarization- and exocytosis-dependent process. In addition, the reserve pool is released by sustained stimulation. In the growth cone the two pools appear to be generated by distinct mechanisms and appear to be constrained to different possible fates, with no evidence for constitutive endosomes entering the releasable (i.e., evoked) pool. It will be of considerable interest to determine whether the evoked and the constitutive endosomes show molecular differences in addition to (or, perhaps, resulting in) their distinct fates.

\section{Functional consequences of two separate endocytotic pools}

The evoked pool shares many properties with the synaptic vesicle pool found in the presynaptic terminal. In fact, it is reasonable to suggest that this evoked pool represents a nascent set of processes in place to allow the growth cone to transform rapidly into a functional presynaptic terminal. This suggestion is made even more likely because the neurons studied here are capable of forming functional synapses with their appropriate targets by the developmental time they are used experimentally. Alternatively, the growth cone could release material [such as neurotransmitters (Hume et al., 1983; Sun and Poo, 1987) or proteases (Kryostosek and Seeds, 1981)] as part of a developmental signaling system that we do not yet understand. It is also intriguing to consider the possibility that local calcium signals within the growth cone could alter growth cone morphology by evoking local membrane addition via calcium-dependent exocytosis. For example, local membrane addition could be involved in filopodial extension or lamellipodial expansion, both of which are likely to be important processes in growth cone pathfinding (O'Connor et al., 1990; Chang et al., 1995; Zheng et al., 1996).

The constitutive pool appears to represent a process unique to the growth cone because such a system has not been reported in the presynaptic terminal. Membrane contributing to elongation might be found within the complex vesicle population found in the growth cone (Cheng and Reese, 1987). The endosomes described here, however, are derived from the growth cone itself; accordingly, other functions seem likely. As an example, both transport away from the growth cone to the cell body and from one site on the growth cone to another are possible. Additionally, the relatively high rates of constitutive endocytosis could permit continual updating of the composition of the growth cone plasma membrane. A redistribution of pumps, channels, and receptors could alter significantly the ability of the growth cone to detect and respond to specific environmental cues.

The interrelationships that endosomes form with cytoskeletal elements and other components of the growth cone will be critical for enabling the functions that these endosomes serve. Whether constitutive and evoked endosomes have different kinds of sub- 
cellular associations and accordingly subserve different functions remains an important question about this system. Investigating the prospective functions and potential additional regulatory mechanisms of these two pools represents a major task in understanding membrane dynamics within the growth cone.

\section{REFERENCES}

Augustine GJ, Burns ME, DeBello WM, Pettit DL, Schweizer FE (1996) Exocytosis: proteins and perturbations. Annu Rev Pharmacol Toxicol 36:659-701.

Bentley D, O'Connor TP (1994) Cytoskeletal events in growth cone steering. Curr Opin Neurobiol 4:43-48.

Betz WJ, Bewick GS (1992) Optical analysis of synaptic vesicle recycling at the frog neuromuscular junction. Science 255:200-203.

Betz WJ, Bewick GS (1993) Optical monitoring of transmitter release and synaptic vesicle recycling at the frog neuromuscular junction. J Physiol (Lond) 460:287-309.

Betz WJ, Mao F, Bewick GS (1992) Activity-dependent fluorescent staining and destaining of living vertebrate motor nerve terminals. J Neurosci 12:363-375.

Betz WJ, Mao F, Smith CB (1996) Imaging exocytosis and endocytosis. Curr Opin Neurobiol 6:365-371.

Bunge MB (1973) Fine structure of nerve fibers and growth cones of isolated sympathetic neurons in culture. J Cell Biol 56:713-735.

Bunge MB (1977) Initial endocytosis of perioxidase or ferritin by growth cones of cultured nerve cells. J Neurocytol 6:407-439.

Chang HY, Takei K, Sydor AM, Born T, Rusnak F, Jay DG (1995) Asymmetric retraction of growth cone filopodia following focal inactivation of calcineurin. Nature 376:686-690.

Cheng TPO, Reese TS (1987) Recycling of plasmalemma in chick tectal growth cones. J Neurosci 7:1752-1759.

Craig AM, Wyborski RJ, Banker G (1995) Preferential addition of newly synthesized membrane protein at axonal growth cones. Nature 375:592-594.

Dai Z, Peng HB (1996) Dynamics of synaptic vesicles in cultured spinal cord neurons in relationship to synaptogenesis. Mol Cell Neurosci 7:443-452.

Dai J, Sheetz MP (1995) Axon membrane flows from the growth cone to the cell body. Cell 83:693-701.

Dailey ME, Bridgman PC (1989) Dynamics of the endoplasmic reticulum and other membranous organelles in the growth cones of cultured neurons. J Neurosci 9:1897-1909.

Dailey ME, Bridgman PC (1993) Vacuole dynamics in growth cones: correlated EM and video observations. J Neurosci 13:3375-3393.

Grimes ML, Beattie E, Mobley WC (1997) A signaling organelle containing the nerve growth factor-activated receptor tyrosine kinase, TrkA. Proc Natl Acad Sci USA 94:9909-9914.

Hemar A, Olivo JC, Williamson E, Saffrich R, Dotti CG (1997) Dendroaxonal transcytosis of transferrin in cultured hippocampal and sympathetic neurons. J Neurosci 17:9026-9034.

Henkel AW, Betz WJ (1995) Monitoring of black widow spider venom (BWSV) induced exo- and endocytosis in living frog motor nerve terminals with FM1-43. Neuropharmacology 34:1397-1406.

Henkel AW, Lubke J, Betz WJ (1996) FM1-43 dye ultrastructural localization in and release from frog motor nerve terminals. Proc Natl Acad Sci USA 93:1918-1923.

Hume RI, Role LW, Fischbach GD (1983) Acetylcholine release from growth cones detected with patches of acetylcholine receptor-rich membranes. Nature 305:632-634.

Kim Y-T, Wu C-F (1987) Reversible blockade of neurite development and growth cone formation in neuronal cultures of a temperaturesensitive mutant of Drosophila. J Neurosci 7:3245-3255.

Kraszewski K, Mundigl O, Daniell L, Verderio C, Matteoli M, De Camilli P (1995) Synaptic vesicle dynamics in living cultured hippocampal neurons visualized with CY3-conjugated antibodies directed against the lumenal domain of synaptotagmin. J Neurosci 15:4328-4342.

Kryostosek A, Seeds NW (1981) Plasminogen activator release at the neuronal growth cone. Science 213:1532-1534.

Kuromi H, Kidokoro Y (1998) Two distinct pools of synaptic vesicles in single presynaptic boutons in a temperature-sensitive Drosophila mutant, shibire. Neuron 20:917-925.

Kuromi H, Kidokoro Y (1999) The optically determined size of exo/ endo cycling vesicle pool correlates with the quantal content at the neuromuscular junction of Drosophila larvae. J Neurosci 19:1557-1565.
Letourneau PC (1996) The cytoskeleton in nerve growth cone motility and axonal pathfinding. Perspect Dev Neurobiol 4:111-123.

Lledo PM (1997) Exocytosis in excitable cells: a conserved molecular machinery from yeast to neuron. Eur J Endocrinol 137:1-9.

Masur SK, Kim YT, Wu CF (1990) Reversible inhibition of endocytosis in cultured neurons from the Drosophila temperature-sensitive mutant shibirets-1. J Neurogenet 6:191-206.

Matteoli M, Haimann C, Torri-Tarelli F, Polak JM, Ceccarelli B, De Camilli P (1988) Differential effect of $\alpha$-latrotoxin on exocytosis from small synaptic vesicles and from large dense-core vesicles containing calcitonin gene-related peptide at the frog neuromuscular junction. Proc Natl Acad Sci USA 85:7366-7370.

Mitchison T, Kirschner M (1988) Cytoskeletal dynamics and nerve growth. Neuron 1:761-772.

Mukherjee S, Ghosh RN, Maxfield FR (1997) Endocytosis. Physiol Rev 77:759-803.

Mundigl O, Ochoa GC, David C, Slepnev VI, Kabanov A, De Camilli P (1998) Amphiphysin I antisense oligonucleotides inhibit neurite outgrowth in cultured hippocampal neurons. J Neurosci 18:93-103.

Murthy VN, Sejnowski TJ, Stevens CF (1997) Heterogeneous release properties of visualized individual hippocampal synapses. Neuron 18:599-612.

O'Connor TP, Duerr JS, Bentley D (1990) Pioneer growth cone steering decisions mediated by single filopodial contact in situ. J Neurosci 10:3935-3949.

Oppenheim RW (1996) The concept of uptake and retrograde transport of neurotrophic molecules during development: history and present status. Neurochem Res 21:769-777.

Overly CC, Hollenbeck PJ (1996) Dynamic organization of endocytic pathways in axons of cultured sympathetic neurons. J Neurosci 16:6056-6064.

Pieribone VA, Shupliakov O, Brodin L, Hilfiker-Rothenfluh S, Czernik AJ, Greengard P (1995) Distinct pools of synaptic vesicles in neurotransmitter release. Nature 375:493-497.

Popov S, Brown A, Poo M-M (1993) Forward plasma membrane flow in growing nerve processes. Science 259:244-246.

Ryan TA, Reuter H, Wendland B, Schweizer FE, Tsien RW, Smith SJ (1993) The kinetics of synaptic vesicle recycling measured at single presynaptic boutons. Neuron 11:713-724.

Schmidt MF, Kater SB (1993) Fibroblast growth factors, depolarization, and substratum interact in a combinatorial way to promote neuronal survival. Dev Biol 158:228-237.

Schmidt MF, Kater SB (1995) Depolarization and laminin independently enable bFGF to promote neuronal survival through different second messenger pathways. Dev Biol 168:235-246.

Schmidt MF, Atkinson PB, Kater SB (1996) Transient elevations in intracellular calcium are sufficient to induce sustained responsiveness to the neurotrophic factor bFGF. J Neurobiol 31:333-344.

Shea TB, Sapirstein VS (1988) Vesicle-mediated delivery of membrane to growth cones during neuritogenesis in embryonic rat primary neuronal cultures. Exp Cell Biol 56:67-73.

Sun Y-A, Poo M-M (1987) Evoked release of acetylcholine from the growing embryonic neuron. Proc Natl Acad Sci USA 84:2540-2544.

Torre E, McNiven MA, Urrutia R (1994) Dynamin 1 antisense oligonucleotide treatment prevents neurite formation in cultured hippocampal neurons. J Biol Chem 269:32411-32417.

Vance JE, Pan D, Vance DE, Campenot RB (1991) Biosynthesis of membrane lipids in rat axons. J Cell Biol 115:1061-1068.

von Gersdorff H, Matthews G (1994) Inhibition of endocytosis by elevated internal calcium in a synaptic terminal. Nature 370:652-655.

Wessells NK, Luduena MA, Letourneau PC, Wrenn JT, Spooner BS (1974) Thorotrast uptake and transit in embryonic glia, heart fibroblasts, and neurons in vitro. Tissue Cell 6:757-776.

Wu LG, Betz WJ (1996) Nerve activity but not intracellular calcium determines the time course of endocytosis at the frog neuromuscular junction. Neuron 17:769-779.

Zagrebelsky M, Buffo A, Skerra A, Schwab ME, Strata P, Rossi F (1998) Retrograde regulation of growth-associated gene expression in adult rat Purkinje cells by myelin-associated neurite growth inhibitory proteins. J Neurosci 18:7912-7929.

Zheng JQ, Wan JJ, Poo MM (1996) Essential role of filopodia in chemotropic turning of nerve growth cone induced by a glutamate gradient. J Neurosci 16:1140-1149. 